\title{
There's more to the working memory capacity-fluid intelligence relationship than just secondary memory
}

\author{
Nash Unsworth, Gene A. Brewer, And Gregory J. Spillers \\ University of Georgia, Athens, Georgia
}

\begin{abstract}
The present study examined the claim that secondary memory processes account for the correlation between working memory capacity and fluid intelligence via a latent variable analysis. In the present study, participants performed multiple measures of secondary memory, working memory capacity, and fluid intelligence. Structural equation modeling suggested that both secondary memory and working memory capacity account for unique variance in fluid intelligence. These results are inconsistent with recent claims that working memory capacity does not account for variance in fluid intelligence over and above what is accounted for by secondary memory. Rather, the results are consistent with models of working memory capacity that suggest that both maintenance and retrieval processes are needed to account for the substantial relation between working memory capacity and fluid intelligence.
\end{abstract}

In a recent study, Mogle, Lovett, Stawski, and Sliwinski (2008) examined the relations among working memory capacity (WMC), secondary memory (SM), and fluid intelligence $(\mathrm{gF})$ via a latent variable analysis. Using a large number of participants and tasks, Mogle et al. found that a latent SM variable was uniquely related to a measure of $\mathrm{gF}$, and that SM accounted for the variance shared between $\mathrm{gF}$ and WMC. Mogle et al. therefore concluded that basic memory processes, such as search and retrieval from $\mathrm{SM}$, account for the $\mathrm{WMC}-\mathrm{gF}$ relation, and that active maintenance of information in the presence of distraction does not.

Mogle et al.'s (2008) data and arguments correspond with much of our previous work, in which we have argued that WMC reflects more than just active maintenance. Specifically, in prior work, we have suggested that WMC is jointly determined by active maintenance in primary memory and by controlled retrieval from SM (Unsworth \& Engle, 2007a), rather than by just SM, as Mogle et al. seemed to suggest. Furthermore - and similar to Mogle and colleagues - we have argued that SM is an important part of the WMC $-\mathrm{gF}$ relationship and that complex spans are not necessarily special, given that other memory tasks can do just as good a job in predicting higher order cognition (Unsworth \& Engle, 2006, 2007a, 2007b). However, we have also argued that SM processes alone cannot fully account for the relation between WMC and gF. This distinction between active maintenance and controlled retrieval is nontrivial, because much prior work has shown that active maintenance in the presence of distraction is a critical component of the predictive power of WMC (Engle
\& Kane, 2004). The notion that SM is the key component does not account for the wealth of data showing a link between WMC and active maintenance in "attention" tasks such as the antisaccade and Stroop tasks. Although we agree with Mogle and colleagues that much can be gained from examining $\mathrm{WMC}$ in the context of more traditional memory models, examining only SM processes severely limits the theoretical and predictive power of WMC. The reason for proposing a dual-component model of WMC was that active maintenance views did not seem to account for the relation between WMC and other memory tasks, and pure memory views of WMC could not account for the relation with attention control tasks. Thus, we proposed that at least two components (active maintenance and controlled retrieval) are needed to fully account for variation in WMC and its relation to gF. Our work suggests that WMC should still account for unique variance in $\mathrm{gF}$ when $\mathrm{SM}$ is controlled for because of shared variance between active maintenance and $\mathrm{gF}$ that is independent of SM.

In their study, Mogle et al. (2008) examined multiple structural equation models in which the latent variables of processing speed, primary memory, WMC, and SM predicted a single measure (Raven Advanced Progressive Matrices) of gF. Mogle et al. found that all of the constructs (except for processing speed) were related to $\mathrm{gF}$. In their final model, Mogle et al. found that SM predicted unique variance in $\mathrm{gF}$, but that WMC did not. Thus, Mogle et al. concluded that SM, but not WMC, was important for $\mathrm{gF}$, contrary to many prior claims. Indeed, Mogle et al. noted that "the major novel result of this study was that SM was a stronger predictor of fluid intelligence than was

N. Unsworth, nunswor@uga.edu 
WMC, which did not account for any variance in fluid intelligence over and above SM" (p. 1075). A close inspection of their correlation matrix, however, suggests that the strong relation between SM and gF may be driven by a single task. Specifically, two of the SM tasks (story recognition and paired associates) correlated with the $\mathrm{gF}$ measure at .26 and .25 (respectively), whereas the word recognition measure correlated appreciably higher, with the gF measure at .39. All of the WMC tasks, however, correlated with gF at .29. Given that only a single indicator of $\mathrm{gF}$ was used, it is possible that the stronger relation between $\mathrm{SM}$ and $\mathrm{gF}$, as compared with that between WMC and $\mathrm{gF}$, was simply the result of the correlation between the gF measure and word recognition.

The aim of the present study was to independently test Mogle et al.'s (2008) claim that SM accounts for the relation between WMC and gF. Given the great deal of work that has been done examining the correlation between WMC and gF (e.g., Ackerman, Beier, \& Boyle, 2005; Kane, Hambrick, \& Conway, 2005), it is critically important to test the claim that the correlation between WMC and $\mathrm{gF}$ rests on nothing more than SM processes. If Mogle et al. are correct, then the SM latent variable should account for unique variance in $\mathrm{gF}$, but WMC should not. On the other hand, if the dual-component model we have suggested is correct, then both SM and WMC should account for unique variance in $\mathrm{gF}$. These competing accounts were tested via a large-scale latent variable analysis in which multiple indicators of each construct were used. Specifically, given the problems with using only a single indicator to represent $\mathrm{gF}$ (i.e., correlations due to idiosyncratic task effects, poor reliability for only a single measure, and narrow definition of the construct of interest), participants in the present study performed the same $\mathrm{gF}$ measure as in the study of Mogle et al. (i.e., the odd items from the Raven Advanced Progressive matrices), as well as two additional $\mathrm{gF}$ tasks that measured both verbal and numerical reasoning (verbal analogies and number series), in order to form a broad gF factor that was not reliant only on spatial reasoning abilities. Additionally, in order to have a broad SM factor, and to ensure that a single SM task did not account for the results, eight total measures of SM-including two measures each of item recognition, source recognition, free recall, and paired associates recall — were used. These tasks were classified as SM tasks, given that the number of to-be-remembered items in each exceeded the theoretical capacity limits of WM and that items had to be remembered after a filled delay (i.e., filled with many other target items or with distractor activity; Cowan, 2008). Finally, three measures of WMC that varied in numerical, visuospatial, and verbal processing (operation, symmetry, and reading span) requirements were used.

\section{METHOD}

\section{Participants}

A total of 173 participants were recruited from the participant pool at the University of Georgia. Participants were between the ages of 18 and 35 and received course credit for their participation. Each participant was tested individually in two laboratory sessions that lasted approximately $2 \mathrm{~h}$ each.

\section{Materials and Procedure}

After signing informed consent, all participants completed operation span, symmetry span, reading span, delayed free recall with semantically related words, item recognition with words, item recognition with pictures, Raven Advanced Progressive Matrices, verbal analogies, number series, and paired associates with word-word pairs in Session 1. In Session 2, all participants completed delayed free recall with unrelated words, paired associates with numberword pairs, gender-source recognition, and picture-source recognition. All tasks were administered in the order listed above.

\section{Tasks}

Operation span (Ospan). Participants solved a series of math operations while trying to remember a set of unrelated letters. Participants were required to solve a math operation, and after solving the operation, they were presented with a letter for $1 \mathrm{sec}$. Immediately after the letter was presented, the next operation was presented. At recall, letters from the current set were recalled in the correct order by clicking on the appropriate letters. For all of the span measures, items were scored if the item was correct and in the correct position. The score was the number of correct items in the correct position.

Symmetry span. Participants were required to recall sequences of red squares within a matrix while performing a symmetryjudgment task. In the symmetry-judgment task, participants were shown an $8 \times 8$ matrix with some squares filled in black. Participants decided whether the design was symmetrical about its vertical axis. The pattern was symmetrical half of the time. Immediately after determining whether the pattern was symmetrical, participants were presented with a $4 \times 4$ matrix with one of the cells filled in red for $650 \mathrm{msec}$. At recall, participants recalled the sequence of redsquare locations in the preceding displays in the order in which they had appeared by clicking on the cells of an empty matrix. The same scoring procedure as that in Ospan was used.

Reading span. Participants were required to read sentences while trying to remember a set of unrelated letters. Participants read a sentence and determined whether it made sense or not. Half of the sentences made sense, whereas the other half did not. Nonsense sentences were made by simply changing one word from an otherwise normal sentence. After participants gave a response, they were presented with a letter for $1 \mathrm{sec}$. At recall, letters from the current set were recalled in the correct order by clicking on the appropriate letters. The same scoring procedure as that in Ospan was used.

Delayed free recall with unrelated words. Participants recalled six lists of 10 words each. All of the words were common nouns that were presented for $1 \mathrm{sec}$ each. After the list presentation, participants engaged in a 16-sec distractor task before recall: Participants saw 10 three-digit numbers appear for $2 \mathrm{sec}$ each, and they were required to write the digits in ascending order. After the distractor task, participants typed as many words as they could remember from the current list in any order they wished. Participants had $45 \mathrm{sec}$ for recall. A participant's score was the total number of items recalled correctly.

Delayed free recall with semantically related words. Participants recalled six lists of 10 words, each broken down into two blocks (three lists per block). All words in each block came from the same semantic category. Following the last word in a list, participants were required to count backward by threes as quickly and as accurately as possible from a three-digit number that was displayed on-screen for $15 \mathrm{sec}$ and to write the numbers down as they proceeded. After the distractor task, participants typed as many words as they could remember from the current list in any order they wished. Participants had $45 \mathrm{sec}$ for recall. A participant's score was the total number of items recalled correctly.

Paired associates with words. Participants were given three lists of 10 word pairs each. All words were common nouns, and the word pairs were presented vertically for $2 \mathrm{sec}$ each. Participants were told that the cue would always be the word on top and that the target would be on bottom. After the presentation of the last word, participants saw the cue word and ??? in place of the target word. Participants were instructed to type in the target word from the cur- 
rent list that matched the cue. Cues were randomly mixed so that the corresponding target words were not recalled in the same order as that in which they had been presented. Participants had $5 \mathrm{sec}$ to type in the corresponding word. A participant's score was the proportion of items recalled correctly.

Paired associates with numbers. This task was exactly the same as the cued recall task with words, but instead of word-word pairs, a random three-digit number served as the cue that was paired with the target word. A participant's score was the proportion of items recalled correctly.

Item recognition with words. In this task, participants were presented with 30 words on-screen at encoding. Words were common nouns presented individually for $2 \mathrm{sec}$ each. At test, participants were presented with 30 old and 30 new words in the center of the screen and were required to indicate whether the word was old or new via a keypress. Participants had $5 \mathrm{sec}$ to press the appropriate key to enter their responses. A participant's score was the proportion of correct responses.

Item recognition with pictures. In this task, participants were presented with 30 pictures on-screen at encoding. Each picture was presented individually for $3 \mathrm{sec}$ each. At test, participants were presented with 30 old and 30 new pictures in the center of the screen and were required to indicate whether the picture was old or new via a keypress. Participants had $5 \mathrm{sec}$ to press the appropriate key to enter their responses. A participant's score was the proportion of correct responses.

Gender source recognition. In this task, participants heard words (30 total words) in either a male or a female voice. Participants were explicitly instructed to pay attention to both the word (item) and the voice the word was spoken in (source). At test, participants were presented with 30 old and 30 new words in the center of the screen. Participants were required to indicate whether the word was new or old and, if old, what voice it had been spoken in, via a keypress. Participants had $5 \mathrm{sec}$ to press the appropriate key to enter their responses. A participant's score was the proportion of correct responses.

Picture source recognition. During the encoding phase, participants were presented with a picture (30 total pictures) in one of four different quadrants on-screen for $1 \mathrm{sec}$. Participants were explicitly instructed to pay attention to both the picture (item) and the quadrant it was located in (source). At test, participants were presented with 30 old and 30 new pictures in the center of the screen. Participants were required to indicate whether the picture was new or old and, if old, in what quadrant it had been presented, via keypress. Participants had $5 \mathrm{sec}$ to press the appropriate key to enter their responses. A participant's score was the proportion of correct responses.

Raven Advanced Progressive Matrices. The Raven (Raven, Raven \& Court, 1998) is a measure of abstract reasoning. The test consists of 36 items presented in ascending order of difficulty. Each item consists of a display of $3 \times 3$ matrices of geometric patterns, with the bottom-right pattern missing. The task for the participant is to select, among eight alternatives, the pattern that correctly completes the overall series. Participants had 10 min to complete the 18 odd-numbered items. A participant's score was the total number of correct solutions.

Verbal analogies. In this task, participants read an incomplete analogy and were required to select the one word out of five possible words that best completed the analogy. Participants had $5 \mathrm{~min}$ to complete 18 test items. A participant's score was the total number of items solved correctly.

Number series. In this task, participants saw a series of numbers and were required to determine what the next number in the series should be. That is, the series followed some unstated rule that participants were required to figure out in order to determine what the next number in the series should be. Participants selected their answer out of five possible numbers that were presented. Participants had 4.5 min to complete 15 test items. A participant's score was the total number of items solved correctly.

\section{RESULTS}

Descriptive statistics and correlations for the measures are shown in Table 1. All of the measures had generally acceptable values of internal consistency, and most of the measures were approximately normally distributed, with values of skewness and kurtosis under the generally accepted values. Note that some of the zero-order correlations and descriptive statistics have been reported in an-

Table 1

Descriptive Statistics and Correlations for All of the Memory and Intelligence Measures

\begin{tabular}{|c|c|c|c|c|c|c|c|c|c|c|c|c|c|c|}
\hline Variable & 1 & 2 & 3 & 4 & 5 & 6 & 7 & 8 & 9 & 10 & 11 & 12 & 13 & 14 \\
\hline 1. Ospan & - & & & & & & & & & & & & & \\
\hline 2. Symspan & .48 & - & & & & & & & & & & & & \\
\hline 3. Rspan & .53 & .38 & - & & & & & & & & & & & \\
\hline 4. Itemrec & .17 & .07 & .16 & - & & & & & & & & & & \\
\hline 5. Picrec & .23 & .09 & .08 & .48 & - & & & & & & & & & \\
\hline 6. Gensour & .11 & .14 & .17 & .28 & .01 & - & & & & & & & & \\
\hline 7. Picsour & .16 & .11 & .04 & .39 & .23 & .24 & - & & & & & & & \\
\hline 8. DFRU & .09 & .05 & .08 & .25 & .19 & .26 & .34 & - & & & & & & \\
\hline 9. DFRR & .23 & .19 & .17 & .30 & .17 & .22 & .21 & .26 & - & & & & & \\
\hline 10. PaW & .17 & .01 & .17 & .24 & .12 & .19 & .27 & .37 & .27 & - & & & & \\
\hline 11. $\mathrm{PaN}$ & .17 & .13 & .12 & .18 & .18 & .26 & .24 & .45 & .19 & .33 & - & & & \\
\hline 12. Raven & .23 & .34 & .22 & .16 & .22 & .16 & .18 & .20 & .18 & .29 & .23 & - & & \\
\hline 13. Analogy & .35 & .23 & .26 & .28 & .26 & .13 & .10 & .12 & .19 & .28 & .06 & .42 & - & \\
\hline 14. NS & .25 & .29 & .22 & .18 & .16 & .04 & .08 & .05 & .15 & .12 & .09 & .20 & .20 & - \\
\hline$M$ & 61 & 31 & 58 & .83 & .96 & .64 & .81 & 34 & 36 & .25 & .13 & 11 & 12 & 10 \\
\hline$S D$ & 12 & 7 & 14 & .09 & .05 & .13 & .13 & 9 & 6 & .22 & .11 & 2 & 3 & 3 \\
\hline Reliability & .73 & .86 & .80 & .67 & .65 & .76 & .83 & .79 & .71 & .83 & .68 & .72 & .80 & .69 \\
\hline Skew & -1.9 & -0.82 & -1.4 & -1.1 & -5.2 & -0.27 & -1.7 & 0.22 & 0.03 & 0.87 & 0.97 & -0.11 & -0.17 & -0.51 \\
\hline Kurtosis & 0.64 & 1.0 & 2.0 & 3.3 & 41.5 & -0.10 & 4.5 & -0.27 & 1.3 & -.35 & 0.37 & -0.21 & -0.42 & -0.12 \\
\hline
\end{tabular}

Note-Ospan, operation span; Symspan, symmetry span; Rspan, reading span; Itemrec, item recognition with words; Picrec, item recognition with pictures; Gensour, gender source recognition; Picsour, picture source recognition; DFRU, delayed free recall with unrelated words; DFRR, delayed free recall with related words; PaW, paired associates with word-word pairs; PaN, paired associates with number-word pairs; Raven, Raven Advanced Progressive Matrices; Analogy, verbal analogies; NS, number series. Reliability was calculated using Cronbach's $\alpha$. Correlations $>.13$ are significant at $p<.05$. 
Table 2

Fit Indices for All Models

\begin{tabular}{lccccccc}
\hline \multicolumn{1}{c}{ Model } & $\chi^{2}$ & $d f$ & $\chi^{2} / d f$ & RMSEA & NNFI & CFI & SRMR \\
\hline Measurement & 116.43 & 74 & 1.57 & .06 & .92 & .94 & .06 \\
Model 1 & 125.97 & 75 & 1.68 & .06 & .91 & .92 & .08 \\
Model 2 & 134.61 & 75 & 1.79 & .07 & .90 & .91 & .08 \\
Model 3 & 116.43 & 74 & 1.57 & .06 & .92 & .94 & .06 \\
Model 3B & 116.49 & 72 & 1.62 & .06 & .92 & .94 & .06 \\
\hline
\end{tabular}

Note-RMSEA, root mean square error of approximation; NNFI, non-normed fit index; CFI, comparative fit index; SRMR, standardized root mean square residual. The $\chi^{2}$ reflects whether there is a significant difference between the observed and reproduced covariance matrices. Therefore, nonsignificant values are desirable. With large sample sizes, even slight deviations can result in a significant value; therefore, the ratio of $\chi^{2}$ to the number of degrees of freedom is also reported. Ratios of two or less indicate acceptable fit. Tests between nested models were examined via a $\chi^{2}$ difference test. The RMSEA and the SRMR both reflect the average squared deviation between the observed and reproduced covariances. The NNFI and the CFI compare the fit of the specified model to a baseline null model. NNFI and CFI values greater than .90 and RMSEA and SRMR values less than .08 are indicative of acceptable fit.

other article (Unsworth \& Brewer, in press). Importantly, none of the analyses or issues discussed in the present article have been reported previously.

A measurement model was specified in which each measure loaded on only its respective construct of interest (i.e., all of the gF measures loaded on the gF construct), and the three constructs (WMC, SM, and gF) were allowed to correlate. As is shown in Table 2, the fit of the model was good. In Figure 1, the resulting model is shown. As can be seen, all tasks loaded significantly on their construct of interest, and all of the latent variables were moderately related to one another.

As in the study of Mogle et al. (2008), we then specified several structural equation models (SEMs) in which WMC and SM latent variables predicted a gF latent variable. In Model 1, only the path from WMC to gF was estimated, and the path from SM to $\mathrm{gF}$ was constrained to 0 . This model suggests that WMC, but not SM, is related to $\mathrm{gF}$. In Model 2, the path from SM to gF was estimated, whereas the WMC to gF path was fixed to 0 . This model suggests that $\mathrm{SM}$, but not $\mathrm{WMC}$, is related to $\mathrm{gF}$, consistent with Mogle et al.'s claim. Finally, in Model 3, both the WMC and SM paths were estimated. This model suggests that both WMC and SM account for unique variance in $\mathrm{gF}$, consistent with a dual-component view. As is shown in Table 2, Model 3 seemed to provide the best fit to the data. In fact, Model 3 fit significantly better than either Model 1 or Model 2 (both $\Delta \chi^{2} \mathrm{~s}>9, p \mathrm{~s}<.01$ ), suggesting that allowing both paths to be freed improved model fit. As is shown in Figure 2A, both WMC and SM accounted for unique variance in $\mathrm{gF}$, and $\mathrm{WMC}$ and SM were correlated. Thus, WMC accounted for variance in $\mathrm{gF}$ over and above what was accounted for by SM, contrary to the results of Mogle et al.

As we have argued, the variance shared between WMC and $\mathrm{gF}$ after controlling for SM likely reflects active maintenance processes that are needed in $\mathrm{gF}$ tasks, independently of SM processes such as search and retrieval. As a further test of this notion, another SEM was specified in which the SM latent variable was composed of the variance common to the eight memory tasks and the three complex spans (the logic being that all WMC and SM tasks are influenced by SM processes). The WMC latent variable was composed of the common variance shared between the complex spans, independent of the shared variance with the memory tasks. The correlation between these two factors was constrained to 0 , and both were allowed to predict gF. This model tests the notion that WMC measures are composed of two separate sources of variance, both of which are related to gF. As is shown in Table 2, the fit of the model (labeled Model 3B) was good. Importantly, as is shown in Figure 2B, both $\mathrm{WMC}$ and SM accounted for unique variance in $\mathrm{gF}$. Thus, WMC accounted for unique variance in $\mathrm{gF}$ that was not accounted for by SM.

\section{DISCUSSION}

The present study examined the claim that the correlation between WMC and gF, which has been found many times previously, is solely due to variation in SM processes (Mogle et al., 2008). Participants performed several gF, WMC, and SM tasks, and the data were examined via several latent variable analyses. The data suggested that an SM latent variable was significantly related to $\mathrm{gF}$ but that, notably, WMC was also related to $\mathrm{gF}$, even after taking SM into account. These results are inconsistent with recent claims that WMC does not account for variance in $\mathrm{gF}$ over and above what is accounted for by SM. Rather, these results are consistent with a dualcomponent model of WMC (Unsworth \& Engle, 2007a), suggesting that at least two sources of variance (active maintenance and controlled retrieval) account for the shared variance between WMC and gF. Thus, contrary to the results of Mogle et al., SM did not account for all of the shared variance between WMC and gF. Rather, WMC and SM accounted for both shared and unique sources of variance in $\mathrm{gF}$.

The present results are also consistent with a number of other models of WMC that suggest that there is more to the 


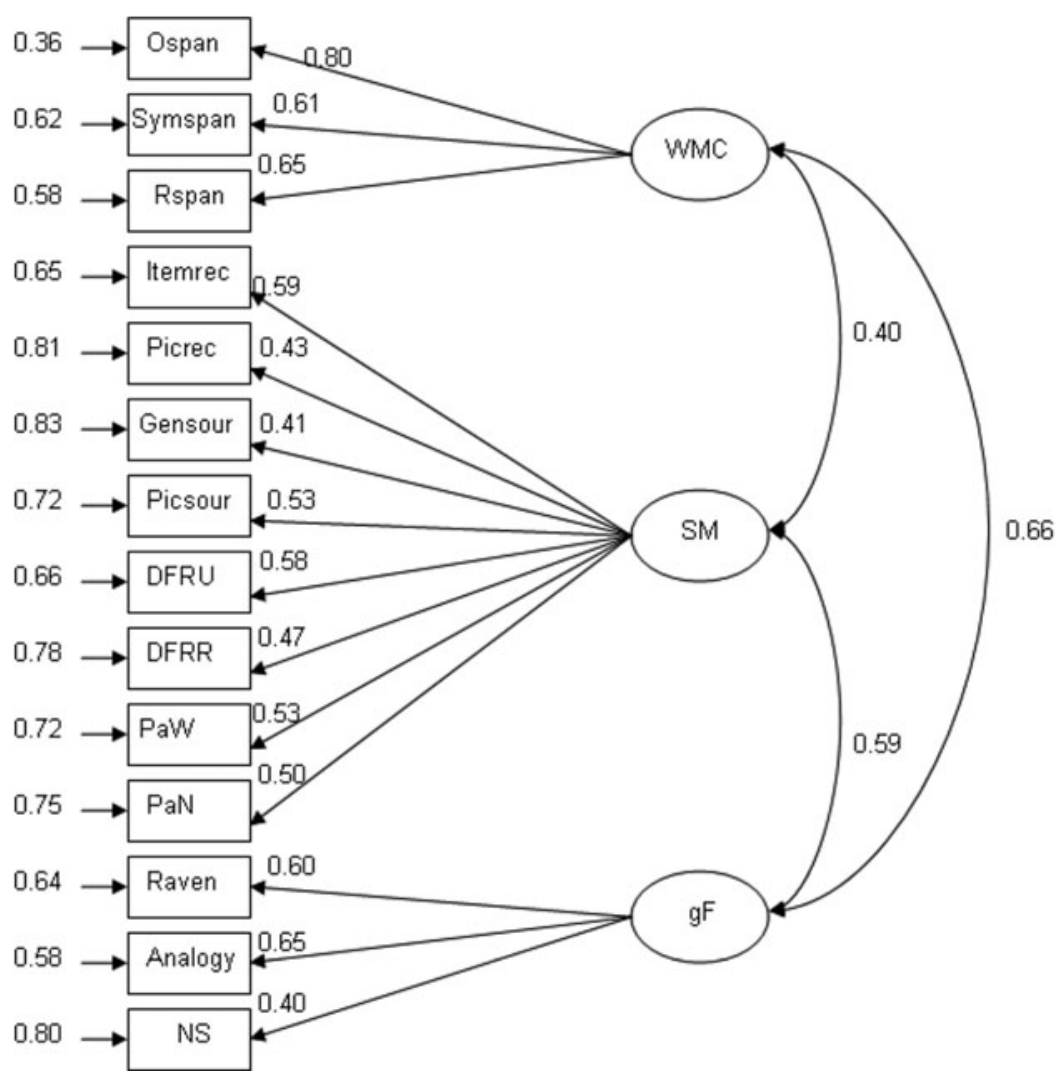

Figure 1. Measurement model for working memory capacity (WMC), secondary memory (SM), and general fluid intelligence (gF). Paths connecting latent variables (circles) to each other represent the correlations between the constructs; the numbers from the latent variables to the manifest variables (squares) represent the loadings of each task onto the latent variable; and numbers appearing next to each manifest variable represent error variance associated with each task. Ospan, operation span; Symspan, symmetry span; Rspan, reading span; Itemrec, item recognition with words; Picrec, item recognition with pictures; Gensour, gender source recognition; Picsour, picture source recognition; DFRU, delayed free recall with unrelated words; DFRR, delayed free recall with related words; PaW, paired associates with wordword pairs; PaN, paired associates with number-word pairs; Raven, Raven Advanced Progressive Matrices; Analogy, verbal analogies; NS, number series. All paths and loadings were significant at the $p<.05$ level.

WMC $-\mathrm{gF}$ relation than just SM. Specifically, the results are consistent with those of Cowan et al.'s (2005) claim that the size or scope of the focus of attention is an important component of individual differences in WMC and is one reason why WMC tasks are related to $\mathrm{gF}$. Cowan et al. demonstrated strong relations between their measures of the size of the focus of attention and $\mathrm{gF}$ measures, even though their size-of-focus tasks did not utilize SM. Consistent with the present results, this suggests that there is substantial shared variance between WMC and $\mathrm{gF}$ that is independent of SM. Additionally, the present results are consistent with Oberauer, Süß, Wilhelm, \& Sander's (2007) claim that the ability to create and maintain bindings in working memory is one reason for individual variation in WMC, and one reason why these tasks correlate so well with gF. Like the present results and those of Cowan et al., this view suggests that processes that are unique to working memory are important for the relation between $\mathrm{WMC}$ and $\mathrm{gF}$. These processes are likely due, in part, to shared variance between WMC and primary memory that is independent of any shared variance with SM (Unsworth \& Engle, 2007b).

Collectively, these results point to the multifaceted nature of WMC, and they suggest that the predictive power of WMC is multiply determined. Some of the predictive power of WMC (in terms of predicting higher order constructs, such as $\mathrm{gF}$ ) is due to variance shared with SM measures, such as free recall, cued recall, and source recognition. At the same time, this is not the whole story. Other sources of variance, such as active maintenance, size of the focus of attention, and short-term binding and maintenance abilities, are also critical to understanding the WMC-gF relation. To truly appreciate the WMC-gF relation, one must look for multiple sources of variance, rather than just a single source. 
A

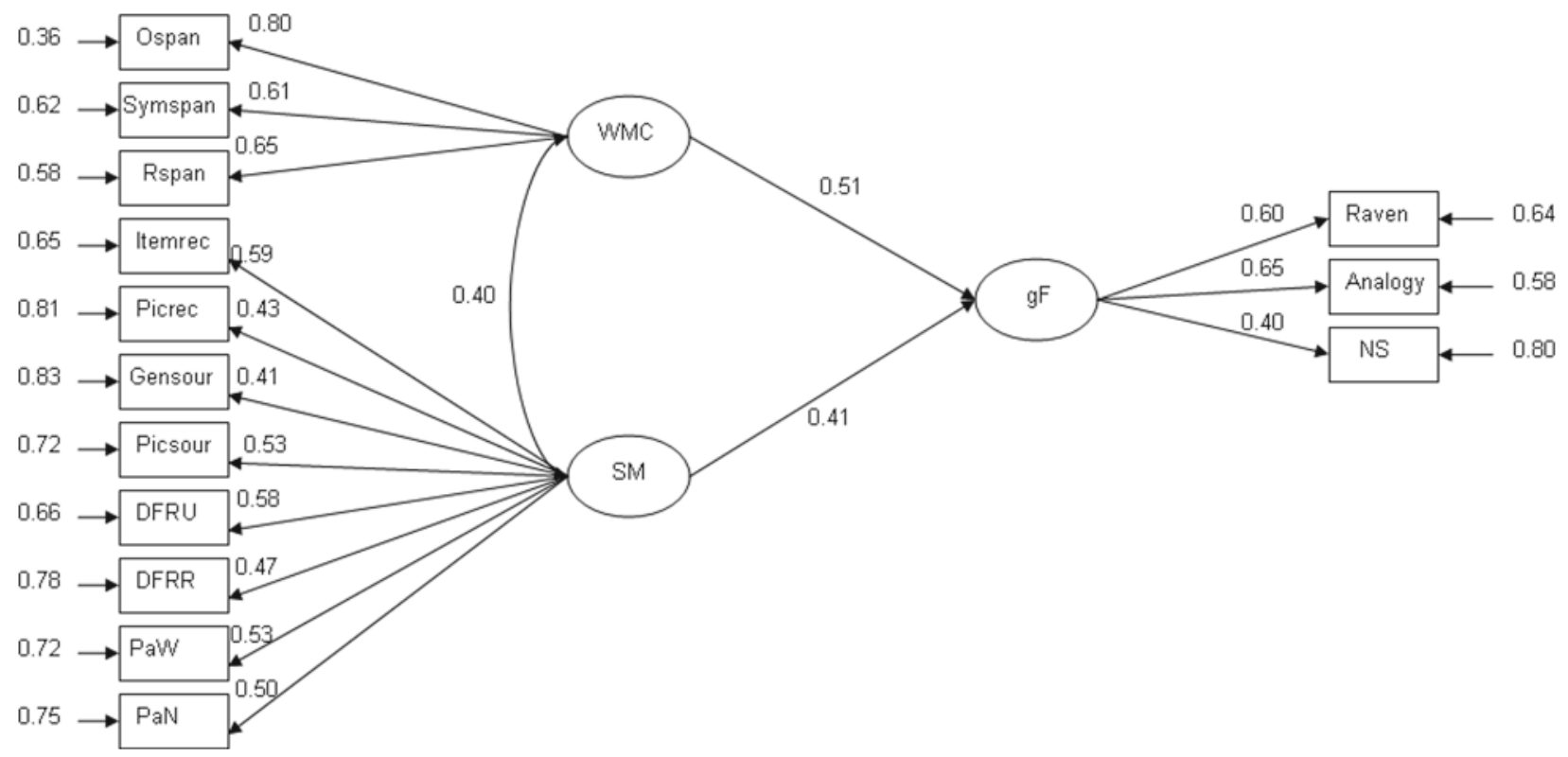

B

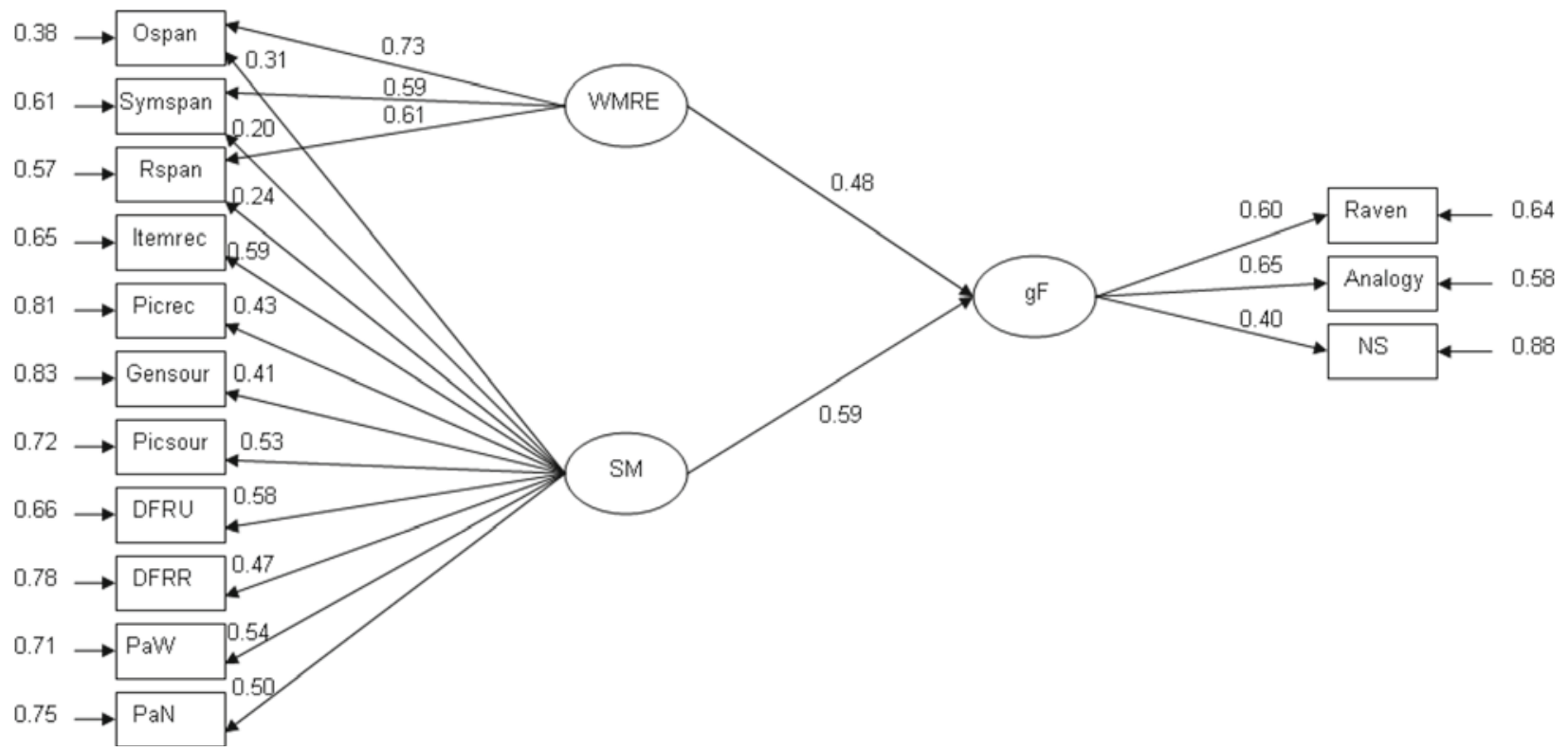

Figure 2. (A) A structural equation model (Model 3) for working memory capacity (WMC) and secondary memory (SM) predicting fluid intelligence (gF). (B) A structural equation model (Model 3B) for the common variance shared across all the memory tasks (SM) and the residual variance common to only the working memory capacity tasks (WMRE) predicting fluid intelligence (gF). All paths and loadings were significant at the $p<.05$ level. See Figure 1 caption for explanation of abbreviations. 


\section{AUTHOR NOTE}

Thanks to Nelson Cowan, Emily Elliott, Mike Kane, and Adam Moore for comments on the manuscript. Correspondence concerning this article should be sent to N. Unsworth, Department of Psychology, University of Georgia, Athens, GA 30602-3013 (e-mail: nunswor@uga.edu).

\section{REFERENCES}

Ackerman, P. L., Beier, M. E., \& Boyle, M. O. (2005). Working memory and intelligence: The same or different constructs? Psychological Bulletin, 131, 30-60.

Cowan, N. (2008). What are the differences between long-term, shortterm, and working memory? In W. Sossin, J.-C. Lacaille, V. F. Castellucci, \& S. Belleville (Eds.), The essence of memory (pp. 323-338). Amsterdam: Elsevier.

Cowan, N., Elliott, E. M., Saults, J. S., Morey, C. C., Mattox, S., Hismuatullina, A., \& Conway, A. R. A. (2005). On the capacity of attention: Its estimation and its role in working memory and cognitive aptitudes. Cognitive Psychology, 51, 42-100.

Engle, R. W., \& Kane, M. J. (2004). Executive attention, working memory capacity, and a two-factor theory of cognitive control. In B. Ross (Ed.), The psychology of learning and motivation (Vol. 44, pp. 145-199). New York: Elsevier.

Kane, M. J., Hambrick, D. Z., \& Conway, A. R. A. (2005). Working memory capacity and fluid intelligence are strongly related constructs: Comment on Ackerman, Beier, and Boyle (2005). Psychological Bulletin, 131, 66-71.
Mogle, J. A., Lovett, B. J., Stawski, R. S., \& SLiwinski, M. J. (2008). What's so special about working memory? An examination of the relationship among working memory, secondary memory, and fluid intelligence. Psychological Science, 19, 1071-1077.

Oberauer, K., Süß, H. M., Wilhelm, O., \& SANDER, N. (2007). Individual differences in working memory capacity and reasoning ability. In A. R. A Conway, C. Jarrold, M. J. Kane, A. Miyake, \& J. N. Towse (Eds.), Variation in working memory (pp. 49-75). New York: Oxford.

Raven, J. C., Raven, J. E., \& Court, J. H. (1998). Progressive matrices. Oxford: Oxford Psychologists Press.

Unsworth, N., \& BREWER, G. A. (in press). Examining the relationships among item recognition, source recognition, and recall from an individual differences perspective. Journal of Experimental Psychology: Learning, Memory, \& Cognition.

Unsworth, N., \& Engle, R. W. (2006). Simple and complex memory spans and their relation to fluid abilities: Evidence from list-length effects. Journal of Memory \& Language, 54, 68-80.

Unsworth, N., \& ENGLE, R. W. (2007a). The nature of individual differences in working memory capacity: Active maintenance in primary memory and controlled search from secondary memory. Psychological Review, 114, 104-132.

Unsworth N., \& EngLe, R. W. (2007b). On the division of short-term and working memory: An examination of simple and complex spans and their relation to higher order abilities. Psychological Bulletin, 133, 1038-1066.

(Manuscript received February 24, 2009; revision accepted for publication May 16, 2009.) 